\title{
On Cosmic-Ray Production Efficiency at Realistic Supernova Remnant Shocks
}

\author{
J. Shimoda*, ${ }^{1}$ T. Inoue ${ }^{2}$, Y. Ohira ${ }^{1}$, R. Yamazaki ${ }^{1}$, A. Bamba ${ }^{1}$ and J. Vink ${ }^{3}$ \\ ${ }^{1}$ Department of Physics and Mathematics, Aoyama-Gakuin University, Sagamihara, Kanagawa \\ 252-5258, Japan \\ ${ }^{2}$ National Astronomical Observatory of Japan, Mitaka, Tokyo 181-8588, Japan \\ ${ }^{3}$ Astronomical Institute Anton Pannekoek/Gravitation and AstroParticle Physics Amsterdam \\ (GRAPPA), University of Amsterdam, Science Park 904, 1098XH Amsterdam, The Netherlands \\ E-mail: s-jiro@phys.aoyama.ac.jp
}

\begin{abstract}
We demonstrate by using three-dimensional magnetohydrodynamics simulations that the cosmicray (CR) production efficiency at supernova remnants (SNRs) is over-predicted if it is based on proper motion measurements of $\mathrm{H} \alpha$ filaments in combination with Rankine-Hugoniot relations. Density fluctuations of upstream medium make shock waves oblique almost everywhere. For oblique shock, the kinetic energy of the shock wave is transferred into that of downstream turbulence as well as thermal energy related to the shock velocity component normal to the shock surface. Our synthetic observations shows that the apparent CR production efficiency as estimated from a lower downstream temperature, depends on the amplitude of density fluctuations of the upstream medium. They are roughly consistent with our analytical estimation of the efficiency. When the upstream medium has density fluctuations of typical diffuse interstellar medium, the apparent CR productions efficiency is observed as $10-40 \%$.
\end{abstract}

The 34th International Cosmic Ray Conference,

30 July- 6 August, 2015

The Hague, The Netherlands

${ }^{*}$ Speaker. 


\section{Introduction}

The energy density of Galactic cosmic rays (CRs) around the Earth is explained if 1-10\% of supernovae explosion energy are goes into CR acceleration. The CR production efficiency at the supernova remnant (SNR) has been widely discussed, which seems to be ubiquitously so high that the background shock structure is modified due to back reaction of CRs. One way to estimate the $\mathrm{CR}$ production efficiency is given by a combination of proper motion measurements of shock front and shocked gas temperature (e.g, $[8,16,7,12,11]$ ). The expansion velocity of a SNR has been measured in various wavelength, from which the downstream temperature $T_{\text {proper }}$ is predicted by using Rankine-Hugoniot relations. If actual downstream temperature $T_{\text {down }}$ can be independently measured, then the CR production efficiency $\eta$ is defined as

$$
\eta=\frac{T_{\text {proper }}-T_{\text {down }}}{T_{\text {proper }}}
$$

where we assume the all missing thermal energy goes into CR acceleration. Note that $\eta$ can be related to $\beta$ which was given by Equation (22) of [17], as $\eta=1-\beta$. Observations of northeastern region of young SNR RCW 86 can be an example. The expansion speed is measured by proper motion of $\mathrm{H} \alpha$ filaments range from 300 to $3000 \mathrm{~km} \mathrm{~s}^{-1}$ with the mean $1200 \mathrm{~km} \mathrm{~s}^{-1}$ [6], while the downstream proton temperature is given by the line width of the broad $\mathrm{H} \alpha$ emission as $T_{\text {down }}=2.3 \mathrm{keV}$ [7] where the observed region containing three bright $\mathrm{H} \alpha$ filaments. The proper motion velocity, $V_{\text {proper }}$, of theses filaments is measured as $1871,1196,1325 \mathrm{~km} \mathrm{~s}^{-1}$, respectively. If these proper motion velocities correspond to the shock velocity locally, we can predict the downstream proton temperature by using Rankine-Hugoniot relations as $T_{\text {proper }} \approx 6.8,2.8,3.4 \mathrm{keV}$, respectively. Then, we obtain $\eta \approx 0.66,0.18,0.32$ for these filaments, which implies highly efficient CR acceleration.

In previous discussions, it was assumed the plane parallel shock wave - that is, the shock normal is parallel to the flow - and that the measured proper motion velocity was identical to the shock velocity. These assumptions could be suitable for spherical symmetric shock wave propagating into homogeneous medium. However, they may not be true for actual SNRs shock waves. Observed proper motions of $\mathrm{H} \alpha$ filaments is dispersed (e.g., [6]), that implies the shock propagation through inhomogeneous medium. At present, it is widely accepted that the interstellar medium (ISM) is highly inhomogeneous (e.g., [2]), in particular, near the young SNRs (e.g, [4, 13]).

We investigated the influence of upstream inhomogeneity which is expected at the realistic diffuse ISM on the above discussion of the CR production efficiency based on three-dimensional magnetohydrodynamics (MHD) simulations ([15]), and simulating $\mathrm{H} \alpha$ filaments whose proper motion is synthetically measured. Our previous results showed that the apparent CR production efficiency, $\eta$, is estimated as 10-40\% in spite of no CR acceleration. In Appendix of [15], a simple analytical argument gives the upper and lower bounds of $\eta$ as

$$
\left(\frac{\Delta \rho}{\langle\rho\rangle_{0}}\right)^{2} \lesssim \eta \lesssim 2 \frac{\Delta \rho}{\langle\rho\rangle_{0}},
$$

where $\Delta \rho /\langle\rho\rangle_{0}$ is the upstream density fluctuation (see Section 3). This bound is roughly consistent with the above numerical results in the case of a typical ISM which has $\Delta \rho /\langle\rho\rangle_{0}=0.3$ at the 
2 pc scale. However, [15] only calculated the case of typical ISM. The environment of actual SNRs has various values of $\Delta \rho /\langle\rho\rangle_{0}$. For example, the young SNR SN 1006 is located far from the galactic plane, which implies that the shock wave may propagate into modestly disturbed medium. Note that several observational results have indicated inhomogeneity of ambient medium around SN 1006([3, 14, 10]). In this paper, we will show the case of $\Delta \rho /\langle\rho\rangle_{0}=0.1$ results by the same way as [15].

\section{Shock Propagation through Inhomogeneous ISM}

Multi-dimensional MHD simulations of shock propagation through inhomogeneous diffuse ISM with Kolmogorov-like density power spectrum have shown that the shock front is rippled due to the fluctuating inertia of the preshock ISM (see [5] for 2D case and [9] for 3D case). Their results strongly suggest that SNR forward shock is locally oblique. For oblique shocks, downstream temperature is given by the velocity component normal to the shock surface $V_{\mathrm{sh}} \cos \theta$ (not shock velocity itself):

$$
k_{\mathrm{B}} T_{\text {down }}=\frac{3}{16} m_{\mathrm{p}} V_{\mathrm{sh}}^{2} \cos ^{2} \theta
$$

where $k_{\mathrm{B}}, m_{\mathrm{p}}$ and $\theta$ are Boltzmann constant, proton mass, the angle between the shock normal and the shock velocity (see Figure 1b of [15]), respectively. The proper motion velocity is identical to the shock velocity component perpendicular to line of sight (LOS). Thus, when the shock front is rippled, the proper motion velocity, $V_{\text {proper }}$, can be larger than $V_{\mathrm{sh}} \cos \theta$ (see, Figure 1a of [15]). Since $V_{\text {proper }} \geq V_{\mathrm{sh}} \cos \theta$, downstream temperature calculated based on the proper motion measurement can be overestimated, i.e., $T_{\text {proper }}=3 m_{\mathrm{p}} V_{\text {proper }}^{2} /\left(16 k_{\mathrm{B}}\right) \geq T_{\text {down }}$, so that $\eta$ is apparently non-zero in spite of no CR acceleration. In the following, using the result of 3D MHD simulation of a shock propagation through an inhomogeneous medium performed by [9], we demonstrated that the above expectation is realized for the typical ISM whose density fluctuations characterized by $\Delta \rho /\langle\rho\rangle_{0}=0.3$ ([15]). In this paper, we show the case of $\Delta \rho /\langle\rho\rangle_{0}=0.1$ to perform that the above argument is generally realized.

\section{Set up of MHD Simulation and Calculation of $\mathrm{H} \alpha$ Emission}

In this paper, we use the data from the simulation performed by [9]. They calculated the shock propagation into realistic diffuse ISM for some cases of the amplitude of density fluctuations. Their Model 3 has the upstream density fluctuation which is given by the Kolmogorov-like isotropic power spectrum $P_{1 \mathrm{D}}(k) \equiv \rho_{k}^{2} k^{2} \propto k^{-5 / 3}$ and $\Delta \rho /\langle\rho\rangle_{0}=0.1$, where $\rho_{k}, k$, and $\langle\rho\rangle_{0}$ are the Fourier component of density, a wave number, the initial mean density and $\Delta \rho$ is the dispersion $\Delta \rho=$ $\left(\left\langle\rho^{2}\right\rangle-\langle\rho\rangle_{0}^{2}\right)^{1 / 2}$. More detailed, see Section 2 of [9]. We regard Model 3 as the case of the shock propagating into modestly disturbed diffuse ISM like the SN 1006 case.

In order to estimate the apparent CR efficiency $\eta$, we calculate the proper motion of $\mathrm{H} \alpha$ emission during 10 years (from $t_{\mathrm{obs}}=700$ to $710 \mathrm{yr}$ ) by the same way as [15]. 


\section{Results of Synthetic observations}

Figure 1 shows the simulated $\mathrm{H} \alpha$ image, and we select 8 regions to measure proper motion. We extract surface brightness profile from these regions and analyze their proper motions by the same way as [6] and [15]. In order to evaluate $\eta$ from Equation 1.1, we calculate $T_{\text {down }}$ as the mean of the downstream proton temperatures of fluid cells just behind the shock surface within the blue box as shown in Figure 1. Table 1 shows the measured proper motion velocity and apparent CR production efficiency $\eta$ for the 8 regions. As expected in Section 2, $T_{\text {proper }}$ is higher than $T_{\text {down }}$ and the efficiency $\eta$ is positive, even through our simulations do not consider the effects of cosmic-ray acceleration. The range of $\eta$ is roughly consistent with our simple analytical estimation given by Equation 1.2.

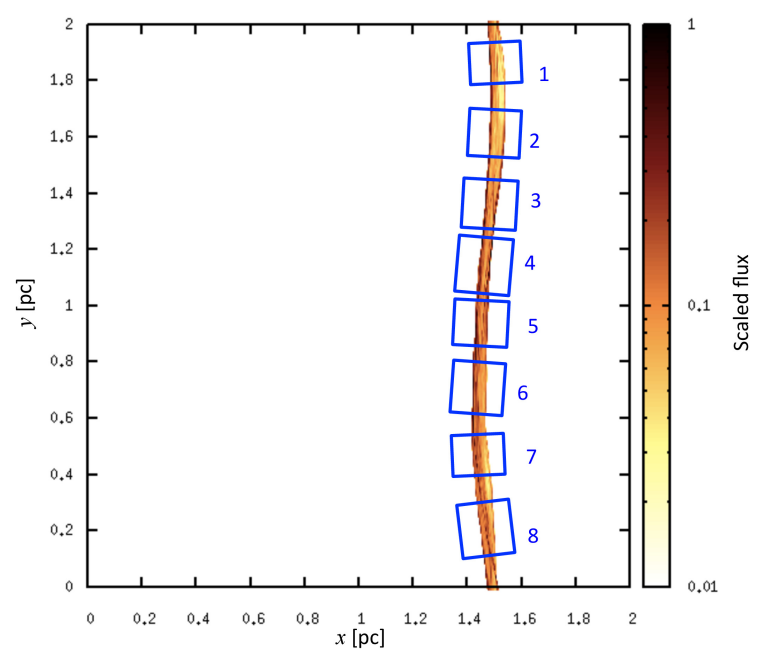

Figure 1: Simulated $\mathrm{H} \alpha$ image. We set the LOS along z-axis. Color represents scaled flux of $\mathrm{H} \alpha$. We selected 8 local regions (blue box), in which proper motion of $\mathrm{H} \alpha$ filament is measured to predict downstream proton temperature.

\begin{tabular}{c|cc|cc} 
Region & $\begin{array}{c}V_{\text {proper }} \\
{\left[10^{8} \mathrm{~cm} \mathrm{~s}^{-1}\right]}\end{array}$ & $\begin{array}{c}T_{\text {proper }} \\
{[\mathrm{keV}]}\end{array}$ & $\begin{array}{c}T_{\text {down }} \\
{[\mathrm{keV}]}\end{array}$ & $\eta$ \\
\hline \hline 1 & $1.64 \pm 0.06$ & $5.3 \pm 0.4$ & 5.0 & $0.05 \pm 0.06$ \\
2 & $1.68 \pm 0.04$ & $5.5 \pm 0.3$ & 4.8 & $0.12 \pm 0.04$ \\
3 & $1.65 \pm 0.05$ & $5.3 \pm 0.3$ & 4.6 & $0.13 \pm 0.05$ \\
4 & $1.61 \pm 0.06$ & $5.0 \pm 0.4$ & 4.5 & $0.11 \pm 0.06$ \\
5 & $1.61 \pm 0.07$ & $5.1 \pm 0.4$ & 4.6 & $0.10 \pm 0.07$ \\
6 & $1.62 \pm 0.12$ & $5.1 \pm 0.8$ & 4.7 & $0.09 \pm 0.12$ \\
7 & $1.66 \pm 0.08$ & $5.4 \pm 0.6$ & 4.9 & $0.09 \pm 0.08$ \\
8 & $1.63 \pm 0.07$ & $5.2 \pm 0.4$ & 4.8 & $0.07 \pm 0.07$ \\
\hline Mean/std. dev. & $1.64 / 0.02$ & $5.2 / 0.1$ & $4.8 / 0.2$ & $0.09 / 0.02$
\end{tabular}

Table 1: Results of proper motion measurements 


\section{Summary}

We have shown that the CR production efficiency $\eta$ seems to be overestimated at the shock wave of SNRs propagating into realistic ISM whose density fluctuations is smaller than typical one if the postshock temperature $T_{\text {proper }}$ is estimated from the proper motion of $\mathrm{H} \alpha$ filaments in combination with Rankine-Hugoniot relation for plane-parallel shock. It may not be suitable assumptions for actual SNR shocks that the shock wave is plane parallel and that the measured proper motion velocity is equivalent to the shock velocity. The rippled, locally oblique shock front is made by the density fluctuations of realistic ISM. For the oblique shocks, the postshock temperature is given not by shock velocity itself but by the velocity component normal to the shock surface $V_{\mathrm{sh}} \cos \theta$ as shown by equation (2.1). Because measurements of proper motion give us velocity component transverse to LOS, the predicted downstream temperature $T_{\text {proper }}$ given by Rankine-Hugoniot relation with the assumption of plane parallel shock is larger than actual downstream temperature $T_{\text {down }}$. Therefore, we claim that the CR production efficiency $\eta$ has some uncertainty, and it can be positive in spite of no CR acceleration. As given by Equation 1.2, our simple analytical estimation of the uncertainty of the apparent CR production efficiency depends on density fluctuations especially $\Delta \rho /\langle\rho\rangle_{0}$. It is roughly consistent to the both cases for $\Delta \rho /\langle\rho\rangle_{0}=0.3$ and 0.1 .

\section{Acknowledgments}

We would like to thank J. C. Raymond for useful comments and suggestions. Numerical computations were carried out on XC30 system at the Center for Computational Astrophysics (CfCA) of National Astronomical Observatory of Japan and K computer at the RIKEN Advanced Institute for Computational Science (No. hp120087). This work is supported by Grant-in-aids from the Ministry of Education, Culture, Sports, Science, and Technology (MEXT) of Japan, No. 15J08894 (J.S.), No. 23740154 (T. I.), No. 248344 (Y. O.), and No. 15K05088 (R.Y.), No. 22684012 (A.B.). T. I. and R. Y. deeply appreciate Research Institute, Aoyama-Gakuin University for helping our research by the fund. R. Y. also thank ISSI (Bern) for support of the team "Physics of the Injection of Particle Acceleration at Astrophysical, Heliospheric, and Laboratory Collisionless Shocks".

\section{References}

[1] J. W. Armstrong, B. J., Rickett \& S. R., Spangler Electron density power spectrum in the local interstellar medium, ApJ, 443, 209-221, 1995.

[2] M. A. de Avillez \& D. Breitschwerdt, The Generation and Dissipation of Interstellar Turbulence: Results from Large-Scale High-Resolution Simulations, ApJ, 665, L35-L38, 2007.

[3] G. M. Dubner et al. The neutral gas environment of the young supernova remnant SN 1006 (G327.6+14.6) A\&A, 387, 1047-1056, 2002.

[4] Y. Fukui, et al. 2003, Discovery of Interacting Molecular Gas toward the TeV Gamma-Ray Peak of the SNR G 347.3-0.5, PASJ, 55, L61-L64, 2003.

[5] J. Giacalone \& J. R. Jokipii, Magnetic Field Amplification by Shocks in Turbulent Fluids, ApJ, 663, L41-L44, 2007. 
[6] E. A. Helder et al., Proper motions of H $\alpha$ filaments in the supernova remnant RCW 86, MNRAS, 435, 910-916, 2013.

[7] E .A. Helder et al., Measuring the Cosmic-Ray Acceleration Efficiency of a Supernova Remnant, Science, 325, 719-722, 2009.

[8] J. P. Hughes, C. E. Rakowski \& A. Decourchelle, Electron Heating and Cosmic Rays at a Supernova Shock from Chandra X-Ray Observations of 1E 0102.2-7219, ApJ, 543, L61-L65, 2000.

[9] T. Inoue et al., The Origin of Radially Aligned Magnetic Fields in Young Supernova Remnants, ApJ, 772, L20, 5pp., 2013.

[10] M. Miceli, M. et al. Shock-Cloud Interaction and Particle Acceleration in the Southwestern Limb of SN 1006 ApJ, 782, L33, 5 pp., 2014.

[11] G. Morlino, P. Blasi, R. Bandiera, \& E. Amato, Cosmic ray acceleration and Balmer emission from SNR 0509-67.5, A\&A, 562, A141, 7pp., 2014.

[12] G. Morlino, P. Blasi, R. Bandiera, \& E. Amato, Cosmic ray acceleration and Balmer emission from $R C W 86$ (G315.4 - 2.3), A\&A, 557, A141, 7pp., 2013.

[13] Y. Moriguchi et al. A Detailed Study of Molecular Clouds toward the TeV Gamma-Ray Supernova Remnant G347.3-0.5, ApJ, 631, 947-963, 2005.

[14] J. C. Raymond et al. The Preshock Gas of SN 1006 from Hubble Space Telescope Advanced Camera for Surveys Observations ApJ, 659, 1257-1264, 2007.

[15] J. Shimoda et al., On Cosmic-ray Production Efficiency at Supernova Remnant Shocks Propagating into Realistic Diffuse Interstellar Medium, ApJ, 803, 98, 6pp., 2015.

[16] V. Tatischeff, \& M. Hernanz, Evidence for Nonlinear Diffusive Shock Acceleration of Cosmic Rays in the 2006 Outburst of the Recurrent Nova RS Ophiuchi, ApJ, 663, L101-L104, 2007.

[17] J. Vink et al. The Relation Between Post-shock Temperature, Cosmic-ray Pressure, and Cosmic-ray Escape for Non-relativistic Shocks, ApJ, 722, 1727-1734, 2010. 